\title{
Robust Estimation of Variable Stiffness in Flexible Joints
}

\author{
Fabrizio Flacco ${ }^{+} \quad$ Alessandro De Luca ${ }^{+}$
}

\begin{abstract}
We consider the problem of estimating on line the nonlinear stiffness of flexible transmissions in robot joints, with special reference to actuation devices with adjustable stiffness in serial configuration. These joints are characterized by a principal motor for controlling the link motion and secondary motor for adjusting the stiffness. In this actuation configuration, the flexible transmission undergoes relatively small deformations and the stiffness estimation problem is more challenging due to poor excitation conditions. We improve our previous general approach, combining a residual-based flexibility torque estimator that uses also a kinematic Kalman filter to handle discretization and quantization errors with an enhanced recursive least squares algorithm that does not suffer from lack of persistent excitation. As a result, stiffness is estimated in a more robust way using only position measurements on the motor sides and motor dynamic parameters. The performance of the proposed estimation method is illustrated through simulations and experiments on the AwAS joint developed at IIT.
\end{abstract}

\section{INTRODUCTION}

In the past, flexibility of the robot transmissions has been considered mainly as an undesired mechanical behavior and a source of control problems [1]. Driven by the recent research in physical Human-Robot Interaction (pHRI), flexibility has been introduced on purpose in the design of robot joints [2], [3]. In particular, the desire of having safer compliant joints while keeping high motion performance in natural and energy efficient robot motion led to the new development of variable stiffness actuation (VSA), where joint stiffness can be varied on the fly during the commanded motion by using two motors. Two different realization of the VSA principle have been mainly investigated. The first consists of using two nonlinear transmissions in agonistic-antagonist arrangement [4]-[6]. Motion and stiffness actuation are then strongly dynamically coupled, and a nonlinear decoupling feedback law is necessary for their simultaneous and accurate control [7]. Conceptually different are the actuators with variable stiffness in serial configuration, in which there is a single flexible transmission whose stiffness can be separately controlled by a secondary motor [8]-[11].

Classical or advanced motion control laws for robots with flexible joints of constant or variable compliance need an accurate knowledge of the joint stiffness [5], [7], [12]. Since there are no available sensors for a direct measure of stiffness, the device stiffness is usually computed from position and/or joint torque sensor data, based on an accurate mathematical model and static calibration procedures. This method is especially critical for VSA-based manipulators,

+ Dipartimento di Informatica e Sistemistica, Università di Roma "La Sapienza", 00185 Roma, Italy \{fflacco,deluca\}@dis.uniroma1.it * Advanced Robotics Lab, Italian Institute of Technology, 16163 Genova, Italy \{irene.sardellitti, nikos.tsagarakis $\} @$ iit.it

\author{
Irene Sardellitti* Nikos G. Tsagarakis*
}

where: i) stiffness should explicitly set under control, ii) the stiffness behavior is intrinsically nonlinear (and possibly time-varying), and iii) its model may be a complex uncertain function of the joint deformation. Therefore, quite recently there has been a growing interest in the development of online stiffness estimation methods.

In [13], a stiffness estimator has been introduced based on the knowledge of the external torque which is explicitly measured by a sensor. The estimator uses an extended Kalman filter to estimate simultaneously the transmission stiffness and the link inertia and damping. However, the estimator uses the time derivative of the measured flexibility torque, and there is an interaction loop between the two used observers leading eventually to noise sensitivity and poor excitation. The stiffness estimators presented in [14], [15] are also based on the knowledge of the flexibility torque and use similarly a joint torque sensor. In [16], we have developed a two-stage stiffness estimator for single flexible transmissions or for double flexible transmissions in antagonist arrangement. In the first stage, the flexibility torque of the transmission is obtained using a residual-based estimator, while in the second stage the stiffness is estimated using either a black-box regressor or a model-based least squares algorithm. Assuming the knowledge of motor inertia and damping, the main merit of this approach is that it requires only the measure of motor position and velocity and of link position, i.e., it uses sensors that are already necessary for control. Moreover, since the stiffness estimator works on the motor side of the flexible transmission, the case of VSA with antagonist arrangements is easily dealt with by having one independent estimator for each transmission and then adding the results to obtain the total device stiffness. Finally, in [17] we proposed a different approach for the second stage where a recursive least squares (RLS) algorithm allows a more efficient and accurate on-line stiffness estimation.

In this paper, we improve both stages of the method presented in [16], [17]. In the first stage, the design of the residual-based estimator of the transmission flexibility torque takes into account also the encoder quantization, one of the most critical problems in the implementation, by using a modified version of the discrete-time kinematic Kalman filter. In the second stage, we consider an enhanced version of the RLS algorithm, based on [18], that improves robustness and ensures convergence of the stiffness estimation also in poor excitation conditions. In fact, we focus our study on the case of actuators with adjustable stiffness [11], where the smaller transmission deformations involved can cause convergence problems. In addition, we need to suitably modify the original stiffness estimation method developed 
for antagonistic VSA so as to cover the case of adjustable stiffness in serial configuration. Figure 1 shows a schematic representation of our stiffness estimator concept for this case.

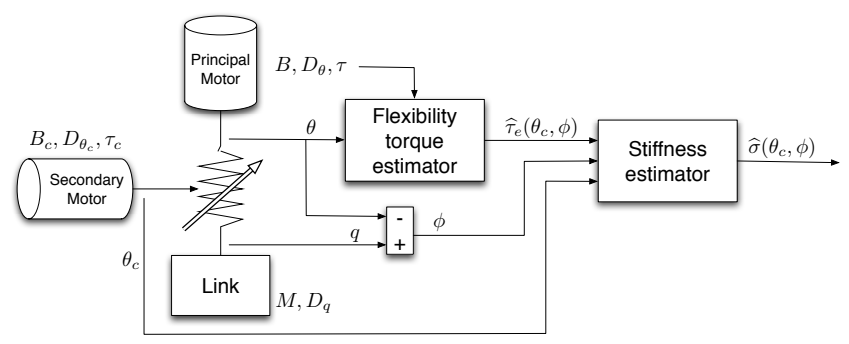

Fig. 1. Two-stage stiffness estimator for an actuator with adjustable stiffness in serial configuration

The paper is organized as follows. The modeling framework is introduced in Sect. II. In Sect. III, we revisit and improve the stiffness estimation method proposed in [16], [17] for a single flexible transmission (and thus also for an antagonistic arrangement of two such transmissions). The modifications needed for estimating the stiffness in joints with adjustable stiffness in serial configuration are presented in Sect. IV. In Sect. V, the problem of a lack of excitation is addressed and a handy solution is provided. Simulation and experimental results for the AwAS device developed at IIT [11] are presented in Sect. VI.

\section{Dynamic Modeling}

Flexible joints are characterized by a deformable transmission that connects the driving motor with the driven link. The deformation $\phi$ of the transmission is the difference between the motor angle $\theta$ and the link angle $q(\phi=q-\theta)$. Being $U_{e}(\phi) \geq 0$ the potential function associated to the deformation $\phi$, with $U_{e}(\phi)=0$ iff $\phi=0$, the flexibility torque across the transmission is $\tau_{e}(\phi)=\partial U_{e}(\phi) / \partial \phi$. We assume that

$$
\tau_{e}(0)=0, \quad \tau_{e}(-\phi)=-\tau_{e}(\phi), \quad \forall \phi,
$$

i.e., no torque is provided through the undeformed transmission, and the transmission has the same behavior in compression and extension.

For a single motor driving a rigid link subject to gravity through a (nonlinear) flexible transmission, the dynamic model takes the form

$$
\begin{aligned}
M \ddot{q}+D_{q} \dot{q}+\tau_{e}(\phi)+g(q) & =\tau_{\mathrm{ext}} \\
B \gamma \ddot{\theta}+D_{\theta} \gamma \dot{\theta}-\frac{1}{\gamma} \tau_{e}(\phi) & =\tau,
\end{aligned}
$$

where $M>0$ and $B>0$ are the link and motor (rotor) inertias, $D_{q} \geq 0$ and $D_{\theta} \geq 0$ are the viscous friction coefficients at the two sides of the transmission, $\gamma>1$ is the gear ratio, $\tau$ is the control torque on the motor axis, and $g(q)$ and $\tau_{\text {ext }}$ are, respectively, the gravity and the environment/disturbance torques acting on the link.
The stiffness of the transmission is defined as the variation rate of the flexibility torque $\tau_{e}(\phi)$ w.r.t. the deformation $\phi$,

$$
\sigma(\phi)=\frac{\partial \tau_{e}(\phi)}{\partial \phi}>0
$$

While slightly different definitions of stiffness can be found in the literature, see e.g., [19], we consider in (4) the internal stiffness of the transmission/flexible joint, usually called passive stiffness. Passive stiffness refers thus to the torque needed to deform the transmission.

The basic dynamic model (2-3) of a single motor-flexible transmission unit is easily modified in order to obtain the model of an actuator with variable stiffness in agonisticantagonistic mode. The two motor-transmission units are modeled will two similar equations of the form (3), one for each motor-transmission with deformation $\phi_{i}=q-\theta_{i}$, for $i=1,2$; in the link equation (2), the sum of the two flexible torques will appear in place of the single $\tau_{e}(\phi)$. Since our estimator works on the motor side, for an antagonistic VSA joint we need simply to use a stiffness estimator for each unit and then sum the resulting estimations [17].

The case of VSA with adjustable stiffness in serial configuration, like the AwAS [11], is more challenging for the estimation process. In this case, the principal motor will control the link position through the flexible transmission while the secondary motor is used to adjust the stiffness by modifying the shape of the potential function associated to the deformation. Stated differently, the position of the secondary motor $\theta_{c}$ will affect the operating point in the flexible transmission characteristics. For its role, we refer to $\theta_{c}$ as the set-point variable, with a slight abuse of terminology. The dynamic model of such class of variable stiffness actuators takes the form

$$
\begin{aligned}
M \ddot{q}+D_{q} \dot{q}+\tau_{e}\left(\theta_{c}, \phi\right)+g(q) & =\tau_{\text {ext }} \\
B \gamma \ddot{\theta}+D_{\theta} \gamma \dot{\theta}-\frac{1}{\gamma} \tau_{e}\left(\theta_{c}, \phi\right) & =\tau \\
B_{c} \gamma_{c} \ddot{\theta}_{c}+D_{\theta_{c}} \gamma_{c} \dot{\theta}_{c}+\frac{1}{\gamma_{c}} \psi_{e}\left(\theta_{c}, \phi\right) & =\tau_{c},
\end{aligned}
$$

where, together with the notations inherited from (2-3), $B_{c}>0$ and $D_{\theta_{c}}>0$ are, respectively, the (rotor) inertia and the viscous friction coefficients of the secondary motor, $\gamma_{c}>1$ is its gear ratio and $\tau_{c}$ its torque (on the motor axis). The function $\psi_{e}\left(\theta_{c}, \phi\right)$ is the coupled flexibility torque, representing how the transmission deformation acts on the secondary motor as a function of its set-point variable. Actually, this is an undesirable dynamic coupling behavior and mechanical solutions that minimize this effect are usually chosen.

For system (5), our goal will be to estimate the stiffness $\sigma\left(\theta_{c}, \phi\right)$ of the transmission without the need of additional sensors beyond the encoders available at both the link and motor sides to measure positions. While velocities of link and motors will be obtained by a suitable numerical filtering of position measures, we will not resort to acceleration measurements (or to further derivation of data) nor to force/torque sensing. 


\section{Stiffness Estimation fOR Single OR Double ANTAGONistic FleXible TRANSMissions}

In this section, the stiffness estimation method proposed in [17] is summarized and some improvements are introduced. With reference to eqs. (2-3), the process is performed in two stages, first by estimating the flexibility torque $\tau_{e}(\phi)$ using a residual-based technique, then by using on line this result to estimate the stiffness $\sigma(\phi)$ with a Recursive Least Squares algorithm based on a general parametric model.

\section{A. Residual for flexibility torque estimation}

To estimate the unmeasured flexibility torque, a residual signal can be generated that provides a filtered version of $\tau_{e}(\phi)$. Denoting as $p=B \dot{\theta}$ the generalized momentum of the motor, the residual is defined as

$$
r_{e}=K_{I}\left(p \gamma+D_{\theta} \gamma \theta-\int_{0}^{t}\left(\tau+r_{e}\right) d s\right),
$$

where $K_{I}>0$ is a free design parameter. From eq. (3), it is easy to check that the residual $r_{e}$ satisfies

$$
\dot{r}_{e}=K_{I}\left(\frac{1}{\gamma} \tau_{e}(\phi)-r_{e}\right),
$$

resulting in a first-order, stable filter of the unknown flexible torque scaled by the gear ratio $\gamma$. A discrete-time implementation of the residual (6) at $t=k T$ is

$$
\begin{aligned}
I_{\tau}(k) & =I_{\tau}(k-1)+\frac{\tau(k)+\tau(k-1)}{2} T \\
r(k) & =K_{I}\left(B \gamma \dot{\theta}(k)+D_{\theta} \gamma \theta(k)-I_{\tau}(k)\right) \\
r_{e}(k) & =\frac{2-T K_{I}}{2+T K_{I}} r_{e}(k-1)+\frac{2(r(k)-r(k-1))}{2+T K_{I}},
\end{aligned}
$$

where $T$ is the sampling time.

It should be noted that the flexibility torque is estimated using only the motor parameters $B$ and $D_{\theta}$, which can be obtained by the motor data sheet. The motor torque $\tau$ is obtained from the known commanded voltage/current, using the motor electrical model and its data sheet. The motor position $\theta$ is measured by an encoder and its velocity $\dot{\theta}$ is obtained numerically. However, the discretization and the presence of encoder quantization are problematic when computing the angular velocity by numerical differentiation.

To address this issue, we propose a modified Kalman filter based on a kinematic model (MKKF). Let $x$ be an angular position and $\dot{x}$ the associated angular velocity. In order to estimate $\boldsymbol{\xi}(k)=(x(k) \dot{x}(k))^{T}$ with a kinematic Kalman filter $(\mathrm{KKF})$, the following system is considered

$$
\begin{aligned}
& \boldsymbol{\xi}(k)=\left(\begin{array}{cc}
1 & T \\
0 & 1
\end{array}\right) \boldsymbol{\xi}(k-1)+\boldsymbol{\mu} \\
& z(k)=\left(\begin{array}{ll}
1 & 0
\end{array}\right) \boldsymbol{\xi}(k)+\nu,
\end{aligned}
$$

where $z(k)$ is the noisy measure (the encoder angle in our case) and the zero mean Gaussian noises $\boldsymbol{\mu}$ and $\nu$ have, respectively, covariance matrix $\boldsymbol{Q}$ and variance $R$. In the state equation (9), acceleration is not considered and $\boldsymbol{\mu}$ represents the noise due also to this absence. Setting $\boldsymbol{\Gamma}=\left(T^{2} / 2 T\right)^{T}$, the covariance matrix of $\boldsymbol{\mu}$ is $\boldsymbol{Q}=V_{a} \boldsymbol{\Gamma} \boldsymbol{\Gamma}^{T}$, where $V_{a}$ is the variance associated to the state. While the variance $R$ of the measures is usually set to a constant value, in the proposed modified KKF it is chosen as a function of the estimated velocity, since the noise due to encoder quantization is significant at low speed and negligible at high speed. In particular, we used

$$
R(k)=\frac{V_{\max }-V_{\min }}{1+e^{\left(|\dot{x}(k)| w_{s}-1\right) \alpha}}+V_{\text {min }},
$$

where $V_{\max }$ and $V_{\min }$ are the maximum and minimum variances considered, $w_{s}=(2 \pi / \Delta) / T$, being $2 \pi / \Delta$ the encoder resolution, and $\alpha$ is a shaping factor (see Sect. VI-B for the resulting effects). In the rest of the paper, whenever we refer to an angular position and/or velocity, we will be considering their evaluation obtained with the MKKF.

\section{B. Stiffness estimation based on Recursive Least Squares}

In this second stage, the flexibility torque $\tau_{e}(\phi)$ is approximated by a nonlinear parametric model function $f(\phi, \boldsymbol{\alpha})$, typically chosen as linear in the unknown $n$-dimensional parameter vector $\boldsymbol{\alpha}$, using a Recursive Least Squares (RLS) algorithm. From measured or computed data, we set the relationship

$$
\widehat{\tau}_{e}(\phi(k))=f(\phi(k), \boldsymbol{\alpha})=\boldsymbol{F}^{T}(k) \boldsymbol{\alpha},
$$

where $\phi(k)$ is the deformation measured at time $t=k T$ and $\widehat{\tau}_{e}(\phi(k))$ may be either the measured flexibility torque, if a joint torque sensor is available, or otherwise its estimate given by the residual $r_{e}(k)$ in eq. (8). The $n$-dimensional vector $\boldsymbol{F}$, i.e., the Jacobian of $f(\phi, \boldsymbol{\alpha})$ w.r.t. the parameter $\boldsymbol{\alpha}$, is usually given by polynomial terms in $\phi$.

The on-line minimization of the sum of the squares of the estimation errors up to time $t=k T$

$$
E(k)=\sum_{i=1}^{k}\left(\widehat{\tau}_{e}(\phi(i))-\boldsymbol{F}^{T}(i) \boldsymbol{\alpha}\right)^{2},
$$

provides the current estimate $\widehat{\boldsymbol{\alpha}}(k)$ by the RLS algorithm as follows:

$$
\begin{aligned}
\epsilon(k) & =\widehat{\tau}_{e}(\phi(k))-\boldsymbol{F}^{T}(k) \widehat{\boldsymbol{\alpha}}(k-1) \\
\rho(k) & =\boldsymbol{F}^{T}(k) \boldsymbol{P}(k-1) \boldsymbol{F}(k) \\
\boldsymbol{K}(k) & =\frac{\boldsymbol{P}(k-1) \boldsymbol{F}(k)}{1+\rho(k)} \\
\widehat{\boldsymbol{\alpha}}(k) & =\widehat{\boldsymbol{\alpha}}(k-1)+K(k) \epsilon(k) \\
\boldsymbol{P}(k) & =\boldsymbol{P}(k-1)-\boldsymbol{K}(k) \boldsymbol{F}^{T}(k) \boldsymbol{P}(k-1) .
\end{aligned}
$$

The algorithm (14), initialized with an a priori estimate $\widehat{\boldsymbol{\alpha}}(0)$ of the parameters and a covariance matrix $\boldsymbol{P}(0)>$ 0 , updates the previous estimation based on the current error $\epsilon(k)$ between the residual/measure and the predicted transmission flexibility torque. The larger the covariance, the larger will be the update of the parameters. Therefore, the covariance matrix $\boldsymbol{P}$ is initialized with large values in case of poor a priori knowledge about the parameters. $\boldsymbol{P}$ typically decreases at each step and there will be no significant parameter updates when the updating factor becomes too 
small. The degree of the polynomial $f(\phi(k), \boldsymbol{\alpha})$ should be adequate for capturing the nonlinearity of the transmission flexibility torque. Otherwise, the RLS algorithm will not track efficiently the data or, even if it converges ( $\boldsymbol{P}$ is small), a non-negligible residual estimation error would result.

Finally, the stiffness estimation is obtained directly from $f(\phi(k), \widehat{\boldsymbol{\alpha}}(k))$ as

$$
\widehat{\sigma}(\phi(k))=\left(\frac{\partial \boldsymbol{F}(k)}{\partial \phi(k)}\right)^{T} \widehat{\boldsymbol{\alpha}}(k) .
$$

For further details on the stiffness estimation for a single or a double antagonistic flexible transmission, we refer to our previous work [17].

\section{Stiffness Estimation for Adjustable STIFFNESS ACTUATORS}

With reference to Fig. 1, consider now an adjustable stiffness actuator modeled by eqs. (5). The estimation of the flexibility torque $\tau_{e}\left(\theta_{c}, \phi\right)$ is obtained using eq. (6) as before, since the principal motor side dynamics is identical and the dependence of $\tau_{e}$ also on $\theta_{c}$ plays no role in the definition of the residual. For stiffness estimation, the RLS algorithm (14) is used but, departing from [17], the linear parameterization of the flexibility torque should be introduced more carefully in order to take into account the dependence from the position of the secondary motor.

We assume that the presence of the secondary motor does not affect assumption (1), or

$$
\tau_{e}\left(\theta_{c}, 0\right)=0, \quad \tau_{e}\left(\theta_{c},-\phi\right)=-\tau_{e}\left(\theta_{c}, \phi\right), \quad \forall \theta_{c}, \phi .
$$

In fact, the set-point variable $\theta_{c}$ of the secondary motor does not change the symmetric nature of the transmission, but will affect the shape of the flexibility torque in a separable way as

$$
\tau_{e}\left(\theta_{c}, \phi\right)=\ell\left(\theta_{c}\right) h(\phi),
$$

i.e., with a positive functional factor that depends on $\theta_{c}$ multiplying the flexibility term due to the deformation $\phi$. A behavior like the one in (17) is highly desirable in the design of VSA systems and is observed in all adjustable stiffness devices we are aware of. As a consequence, in the parametric approximation of $\tau_{e}$, we will consider the following two polynomials for $h(\phi)$ and $\ell\left(\theta_{c}\right)$ :

$$
\begin{aligned}
h(\phi, \boldsymbol{\alpha}) & =\sum_{i=0}^{n-1} \alpha_{i} \phi^{2 i+1}, \\
\ell\left(\theta_{c}, \boldsymbol{\beta}\right) & =\sum_{j=0}^{m-1} \beta_{j} \theta_{c}^{j} .
\end{aligned}
$$

For the sake of simplicity we do not force positivity of the factor $\ell\left(\theta_{c}\right)$, which would lead to the need of adding constraints in the RLS algorithm without effective improvement of the estimate. In the estimation process, we do not need a separate (and non trivial) estimation of the $n+m$ unknown parameters in vectors $\boldsymbol{\alpha}$ and $\boldsymbol{\beta}$, but we linearly re-parameterize the problem in terms of the $n \cdot m$ scalar parameters

$$
\eta_{i, j}=\alpha_{i} \beta_{j}
$$

Based on eq. (17), the function $f$ fitting $\tau_{e}$ will thus be

$$
f\left(\theta_{c}, \phi, \boldsymbol{\eta}\right)=\sum_{i=0}^{n-1} \sum_{j=0}^{m-1} \eta_{i, j} \theta_{c}^{j} \phi^{2 i+1}=\boldsymbol{F}^{T}\left(\theta_{c}, \phi\right) \boldsymbol{\eta} .
$$

The Jacobian of $f\left(\theta_{c}, \phi, \boldsymbol{\eta}\right)$ w.r.t. the new parameter vector $\boldsymbol{\eta}$ is given by

$$
\begin{aligned}
& \boldsymbol{F}^{T}=\left(\begin{array}{llll}
\phi & \phi^{3} & \ldots & \phi^{2 n-1}
\end{array}\right. \\
& \theta_{c} \phi \quad \theta_{c} \phi^{3} \quad \ldots \quad \theta_{c} \phi^{2 n-1} \\
& \begin{array}{llll}
\ldots & \ldots & \ldots & \ldots
\end{array} \\
& \left.\theta_{c}^{m-1} \phi \quad \theta_{c}^{m-1} \phi^{3} \quad \ldots \quad \theta_{c}^{m-1} \phi^{2 n-1}\right) .
\end{aligned}
$$

Considering a discrete-time implementation, the estimation of the unknown parameter $\widehat{\boldsymbol{\eta}}$ is obtained using (14), where $\widehat{\boldsymbol{\alpha}}(k)$ is obviously replaced by $\widehat{\boldsymbol{\eta}}(k)$. Therefore, the stiffness estimate at time $t=k T$ is given again by the relation (15) suitably modified, i.e.,

$$
\begin{aligned}
\widehat{\sigma}\left(\theta_{c}(k), \phi(k)\right) & =\frac{\partial f\left(\theta_{c}(k), \phi(k)\right)}{\partial \phi(k)}=\left(\frac{\partial \boldsymbol{F}(k)}{\partial \phi(k)}\right)^{T} \widehat{\boldsymbol{\eta}}(k) \\
& =\sum_{i=0}^{n-1} \sum_{j=0}^{m-1}(2 i+1) \widehat{\eta}_{i, j}(k) \theta_{c}^{j}(k) \phi^{2 i}(k) .
\end{aligned}
$$

\section{Handling Poor Excitation Conditions}

For a robust parameter estimation, it is important that the input signal changes sufficiently so that the collected data contain enough information about the characteristics of the function to be estimated. This is related to the concept of persistent excitation of signals. Unlike the case of antagonistic VSA, where the deformation of the two transmissions are significant in order to simultaneously control both link motion and stiffness, in actuators with adjustable stiffness the deformation is minimal, because stiffness is controlled using the secondary motor. Therefore, in this case the persistent excitation requirement is a very critical issue for stiffness estimation. Since the RLS estimation may become unstable in the presence of poor excitation, we propose a modified RLS based on the results of [18], which are briefly recalled.

Assume that measured data $y$ are generated at $t=k T$ as

$$
y(k)=\boldsymbol{F}^{T}(k) \overline{\boldsymbol{\eta}},
$$

where $\overline{\boldsymbol{\eta}}$ is the true but unknown parameter vector. In the parameter estimation error $\widetilde{\boldsymbol{\eta}}=\widehat{\boldsymbol{\eta}}-\overline{\boldsymbol{\eta}}$, it is possible to discriminate a component $\widetilde{\boldsymbol{\eta}}_{U}$ that belongs to the so-called unexcitation subspace and a component $\widetilde{\boldsymbol{\eta}}_{E}$ that belongs to its orthogonal complement $\Omega_{E}=\Omega_{U}^{\perp}$, the excitation subspace. Consider the following modification to the RLS algorithm (14) used for the estimate $\widehat{\boldsymbol{\eta}}$

$$
\begin{aligned}
\widehat{\boldsymbol{\eta}}(k) & =\widehat{\boldsymbol{\eta}}(k-1)+a(k) \boldsymbol{K}(k) \epsilon(k) \\
\boldsymbol{P}(k) & =\boldsymbol{P}(k-1)-a(k) \boldsymbol{K}(k) \boldsymbol{F}^{T}(k) \boldsymbol{P}(k-1),
\end{aligned}
$$

where the scalar $a(k)$ is a time-varying function. 
Under the data generation assumption (24), it has been proven in [18] that, if there exists a scalar $c>0$ such that

$$
\frac{a(k)}{1+\rho(k)-a(k) \rho(k)} \geq c, \quad \forall k,
$$

then, for every given $\widehat{\boldsymbol{\eta}}(0)$ and $\boldsymbol{P}(0)>0$ :

1) $\|\widetilde{\boldsymbol{\eta}}(k)\| \leq \lambda, \quad \forall k$, being $\lambda$ a suitable constant;

2) $\lim _{k \rightarrow \infty} \widetilde{\boldsymbol{\eta}}_{E}(k)=\mathbf{0}$.

Provided that $a(k)$ can be chosen so as to verify condition (26), we obtain thus practical convergence of the parameter estimation even in poor excitation cases. Given a constant $c>0$, the stability factor $a(k)$ is then simply chosen as

$$
a(k)=\frac{c+c \rho(k)}{1+c \rho(k)} .
$$

Summarizing, the enhanced RLS algorithm is obtained from (14), with $\widehat{\boldsymbol{\eta}}$ in place of $\widehat{\boldsymbol{\alpha}}$, by replacing the last two equations therein with (25) and using (27).

\section{Simulations And Experiments on the AwAS}

The stiffness estimation method has been tested through simulations and experiments, using the Actuator with Adjustable Stiffness (AwAS) developed by the Italian Institute of Technology (IIT) and presented in [11]. The relative simplicity of the potential function associated to the transmission deformation of the AwAS and the presence of a sensor to measure the flexibility torque, which allows a validation of the results, makes this actuator a useful benchmark for testing our stiffness estimator.

\section{A. Modeling the AwAS}

The AwAS (Fig. 2) actuator adjusts the stiffness at the joint through the variation of the relative distance between a pair of springs and the center of rotation of the joint, using a lever mechanism. The dynamics of the AwAS is described

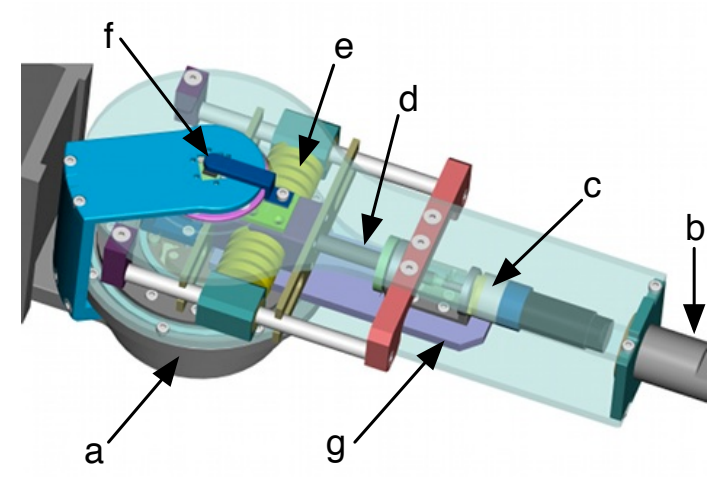

Fig. 2. CAD model of the Actuator with Adjustable Stiffness (AwAS): the principal motor (a) adjusts the link (b) positioning; the secondary motor (c) drives a ballscrew mechanism (d), which moves the relative position of a pair of antagonistic springs (e) with respect to the center of rotation of the joint (f); the springs connect both the output link (b) and the intermediate link $(\mathrm{g})$, which in turn is connected with the principal motor by model (5), where the flexibility torque $\tau_{e}(r, \phi)$ and the stiffness $\sigma(r, \phi)$ are defined by

$$
\tau_{e}(r, \phi)=k_{s} r^{2} \sin 2 \phi
$$

and

$$
\sigma(r, \phi)=2 k_{s} r^{2} \cos 2 \phi,
$$

being $k_{s}$ the stiffness of the springs and $r$ the length of the lever arm, which is the effective distance between the center of rotation of the joint and the springs. The lever arm is adjusted by the secondary motor as

$$
r=r_{0}-b \theta_{c},
$$

where $r_{0}$ is the initial length of the lever arm, $\theta_{c}$ is the angular position of the motor, and $b$ is the transmission ratio between the secondary motor and the ballscrew. Finally, the torque that the transmission applies back to the secondary motor is

$$
\psi_{e}(r, \phi)=-2 k_{s} b r \sin ^{2} \phi .
$$

We note that the lever arm $r$ obtained from (30) can be directly used in place of $\theta_{c}$ in the enhanced RLS estimation algorithm. With reference to the model (5) and eqs. (28-31), the nominal parameters of the AwAS are:

$$
\begin{aligned}
& M=0.1, B=2.3 \cdot 10^{-5}, B_{c}=1.29 \cdot 10^{-7}\left[\mathrm{~kg} \cdot \mathrm{m}^{2}\right], \\
& D_{q}=0.15, D_{\theta}=0.001, D_{\theta_{c}}=0.0141[\mathrm{~N} \cdot \mathrm{m} \cdot \mathrm{s} / \mathrm{rad}], \\
& \gamma=50, \gamma_{c}=23, k_{s}=14220[\mathrm{~N} / \mathrm{m}], r_{0}=0.1[\mathrm{~m}], \\
& b=0.0025 / 2 \pi[\mathrm{m} / \mathrm{rad}] .
\end{aligned}
$$

\section{B. Simulation results}

The estimator has been tested first in realistic simulations performed with Simulink. The AwAS model is represented by (5), with the parameters of Sect. VI-A. We have included in the simulations the presence of encoders with $\Delta=40000$ pulses/turn for measuring $q$ and $\theta$, and with $\Delta_{c}=1024$ for $\theta_{c}$. We considered also the effect of noise added to the motor torque $\tau$ used in the residual computation (6) as well as to the sensed torque $\tau_{e}$, which can be used alternatively to the residual as input $\widehat{\tau}_{e}$ to the RLS algorithm. A tilda denotes the noisy signals, i.e.,

$$
\begin{aligned}
\widetilde{\tau} & =\tau+\nu_{\tau}, \\
\widetilde{\tau}_{e}\left(\theta_{c}, \phi\right) & =\tau_{e}\left(\theta_{c}, \phi\right)+\nu_{\tau_{e}},
\end{aligned}
$$

where $\nu_{\tau}$ and $\nu_{\tau_{e}}$ are zero mean gaussian noises with variance $10^{-5}$ and $10^{-3}$, respectively.

To show the robustness of our algorithm, we performed the stiffness estimation in three modalities, using three different signals as input $\widehat{\tau}_{e}$ of the RLS algorithm. In the MODEL modality, we used as input the flexibility torque obtained from eq. (28) with the nominal system data ${ }^{1}$. In the SENSOR modality, we used as input the flexibility torque measured by a noisy joint torque sensor, see eq. (33). Finally, in the RESIDUAL modality we fed the RLS algorithm with the residual that estimates the flexibility torque. In this last

\footnotetext{
${ }^{1}$ In simulations, this flexibility torque is indeed also the actual one.
} 
modality, the residual (6) has been evaluated using the principal motor position $\theta$ and velocity $\dot{\theta}$ obtained by processing the encoder data with the MKKF presented in Sec. III-A, as well as the noisy motor torque (32). Summarizing, for the three modalities we have:

$$
\begin{aligned}
& \widehat{\tau}_{e}\left(\theta_{c}, \phi\right)=\tau_{e}\left(\theta_{c}, \phi\right) \quad \text { MODEL } \\
& \widehat{\tau}_{e}\left(\theta_{c}, \phi\right)=\widetilde{\tau}_{e}\left(\theta_{c}, \phi\right) \quad \text { SENSOR } \\
& \widehat{\tau}_{e}\left(\theta_{c}, \phi\right)=\gamma r_{e} \quad \text { RESIDUAL }
\end{aligned}
$$

The simulations were run with sampling time $T=1 \mathrm{~ms}$, using as torque inputs for the principal and secondary motor $\tau=0.3 \sin 0.2 \pi$ and $\tau_{c}=8 \sin 0.2 \pi[\mathrm{Nm}]$, from the initial configuration $q(0)=\theta(0)=0, \theta_{c}(0)=-30 / b$ [rad] and with the system at rest. The chosen MKKF parameters in eq. (11) were $V_{\max }=10, V_{\min }=0.1$, and $\alpha=6$, obtained by considering a nominal minimum and maximum velocity which rely on a minimum and maximum variance. The remaining estimation parameters were: $K_{I}=300$, in eq. (6), which represent the filter bandwidth of the residual; $\boldsymbol{P}(0)=10^{20} \boldsymbol{I}_{n \times m}$ in eq. (14); $n=6$ and $m=3$ in eqs. (18) and (19); and $c=10^{-20}$ in eq. (27). Therefore, the parameter vector $\boldsymbol{\eta}$ has dimension $n \cdot m=18$, and we set $\widehat{\boldsymbol{\eta}}(0)=\mathbf{0}$. The orders of the polynomial functions $m$ and $n$ are selected on the basis of the nominal model. However, higher orders can be selected to better fit the nonlinearities.

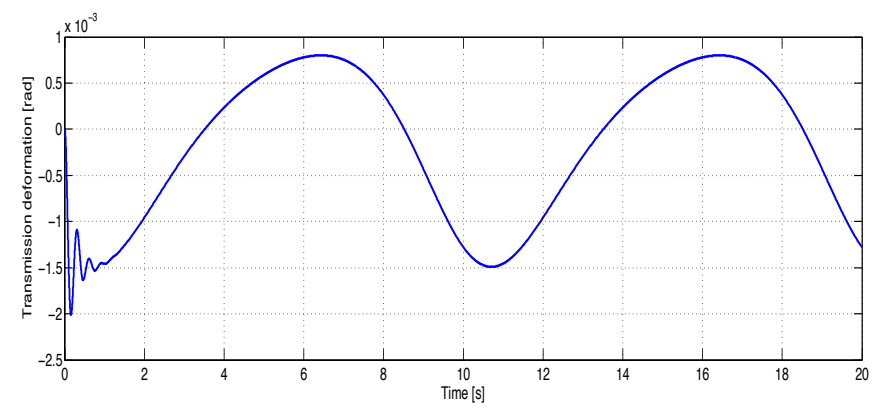

Fig. 3. Transmission deformation $\phi$ in simulation

Figure 3 shows the time behavior of the transmission deformation $\phi$ during the simulation. Note that its maximum value is very small (of the order $0.001 \mathrm{rad}$ ) and we are thus in a situation of very poor excitation for the RLS algorithm.

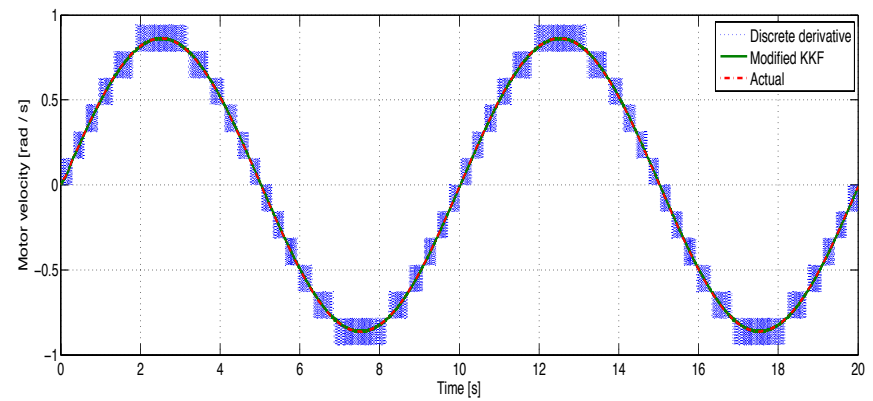

Fig. 4. Motor angular velocity $\dot{\theta}$ : Actual (dot-dashed, red), obtained with numerical differentiation (dotted, blue), estimated with MKKF (solid, green)

The need for MKKF processing of the encoder data follows from the results in Fig. 4. The principal motor velocity $\dot{\theta}$ obtained by numerical differentiation of the encoder position data suffers from the presence of quantization.

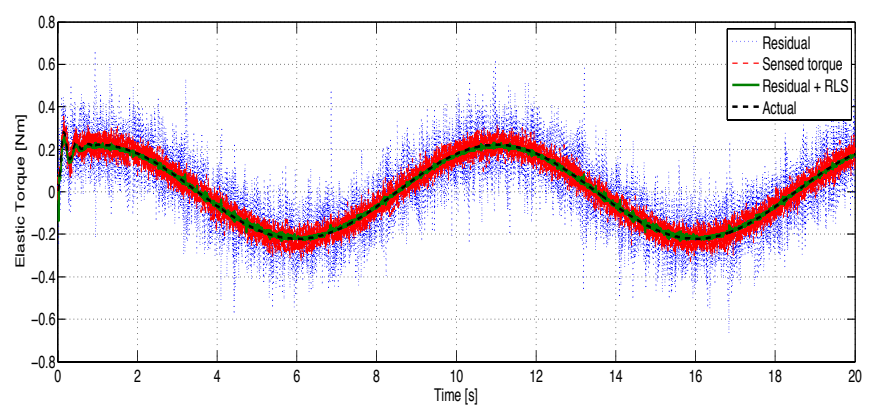

Fig. 5. Flexibility torque: Actual $\tau_{e}\left(\theta_{c}, \phi\right)$ (dashed, black), estimated from the residual as $\gamma r_{e}$ (dotted, blue), measured by a noisy torque sensor $\widetilde{\tau}_{e}\left(\theta_{c}, \phi\right)$ (dashed, red), obtained from RLS algorithm with residual input as $f\left(\theta_{c}, \phi, \widehat{\boldsymbol{\eta}}\right)$ (solid, green)

Figure 5 compares two different estimations and the noisy measure of the flexibility torque $\tau_{e}\left(\theta_{c}, \phi\right)$ with its actual (nominal) evolution. In particular, both the measured torque and the estimation of the flexibility torque obtained directly from the residual (6) are quite noisy under the assumed operative conditions. However, a reliable (filtered and centered) flexibility torque estimation is obtained when feeding the residual into the RLS algorithm and using the estimated parameter vector $\widehat{\boldsymbol{\eta}}$ in eq. (21).

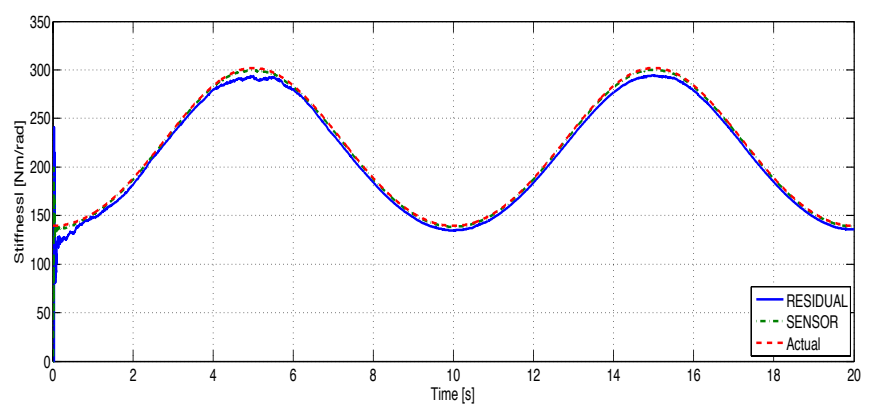

Fig. 6. Stiffness $\sigma\left(\theta_{c}, \phi\right)$ : Actual (dashed, red), SENSOR estimation (dotdashed, green), RESIDUAL estimation (solid, blue) [MODEL and SENSOR estimation practically overlap, so the former is not shown]

Finally, the time evolutions of the stiffness estimated in the different modalities are reported in Fig. 6, compared to the actual evolution. To quantify the different stiffness estimation, we have considered two performance indices, namely the mean square error (MSE) and the mean square relative error percentage (MSREP) over $p$ samples,

$$
\begin{aligned}
\text { MSE } & =\frac{\sum_{k=0}^{p}\left[(\sigma(k)-\widehat{\sigma}(k))^{2}\right]}{p}, \\
\operatorname{MSREP} & =\frac{\sum_{k=0}^{p}\left[\left(\frac{\sigma(k)-\widehat{\sigma}(k)}{\sigma(k)}\right)^{2}\right]}{p} 100 .
\end{aligned}
$$

From the results in Tab. I, all methods perform similarly well. Being these simulation results, the MODEL stiffness estimate is indeed very accurate despite the presence of 
TABLE I

PERFORMANCE OF STIFFNESS ESTIMATION IN SIMULATION

\begin{tabular}{|c||c|c|}
\hline Estimation & MSE & MSREP \\
\hline MODEL & 24.1365 & $0.12 \%$ \\
\hline SENSOR & 24.3630 & $0.12 \%$ \\
\hline RESIDUAL & 128.1614 & $0.58 \%$ \\
\hline
\end{tabular}

encoder quantization. The SENSOR estimation is also very accurate, confirming the benefit of using anyway a processing by the enhanced RLS algorithm. The RESIDUAL estimation has a higher MSE, due to the propagation of input noise through the two stages of the estimation process; however, its MSREP is still less than $1 \%$ which allows us to state that torque sensing seems not strictly needed.

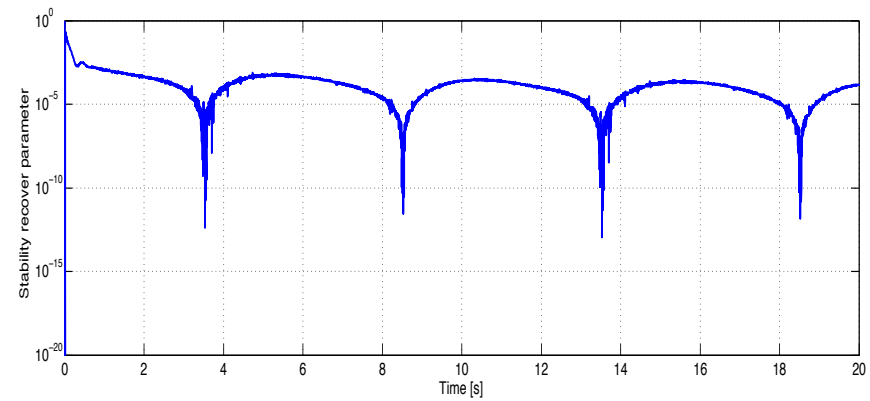

Fig. 7. Stability factor $a(k)$ in the RESIDUAL estimation method (semilogarithmic scale)

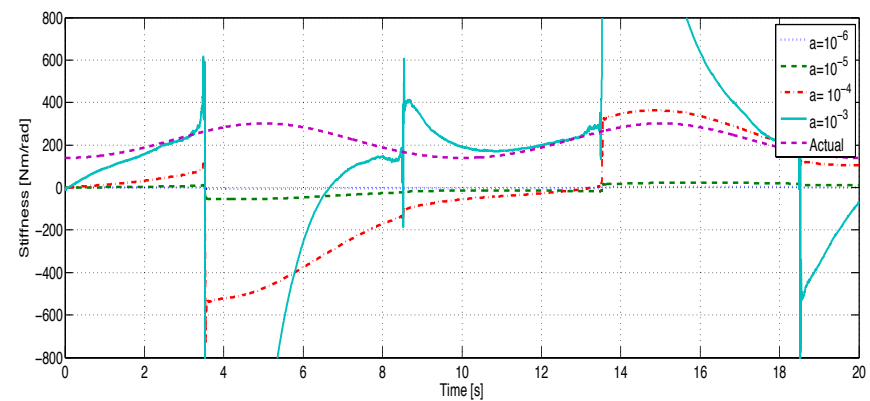

Fig. 8. Stiffness estimated by the RESIDUAL method, for different but constant stability factors

The last issue analyzed in simulation concerns the use of the stability factor $a(k)$ of eq. (27) for stiffness estimation by the RESIDUAL method. The time evolution of this factor is shown on a semi-logarithmic scale in Fig. 7. By comparing this with Fig. 3, one can see that the low peaks of $a(k)$ are associated to values of the transmission deformation close to zero. In these cases, the Jacobian $\boldsymbol{F}$ contains very small values, which imply a poor excitation for the estimator. If a constant value were used for the stability factor, we would obtain two possible behaviors: with a relatively small stability factor, the stiffness estimation is not affected by poor excitation but the convergence is very slow; with a large stability factor, the convergence is faster but the estimation is very sensitive to poor excitation conditions. These behaviors are shown in Fig. 8, justifying the relevance of using the enhanced RLS algorithm with time-varying stability factor.

\section{Experimental results}

In the experiments, the principal and secondary motors drive the AwAS with sinusoidal torque signals. The obtained transmission deformation and the lever arm position are reported in Fig. 9 and Fig. 10, respectively.

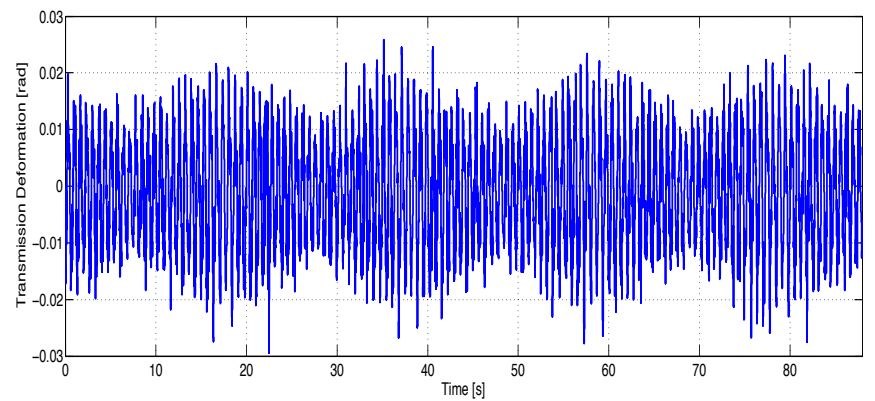

Fig. 9. Transmission deformation $\phi$ in experiment

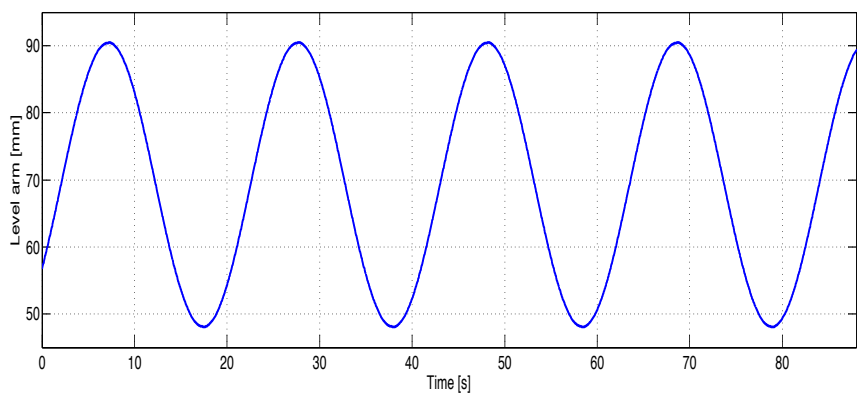

Fig. 10. Level arm position $r$ in experiment

To validate our estimation algorithm, we take advantage of the calibrated torque sensor available in the AwAS and consider the flexibility torque measured by the torque sensor as the ground truth. The actual difference between the nominal flexibility torque and its measure by the torque sensor is shown in Fig. 11.

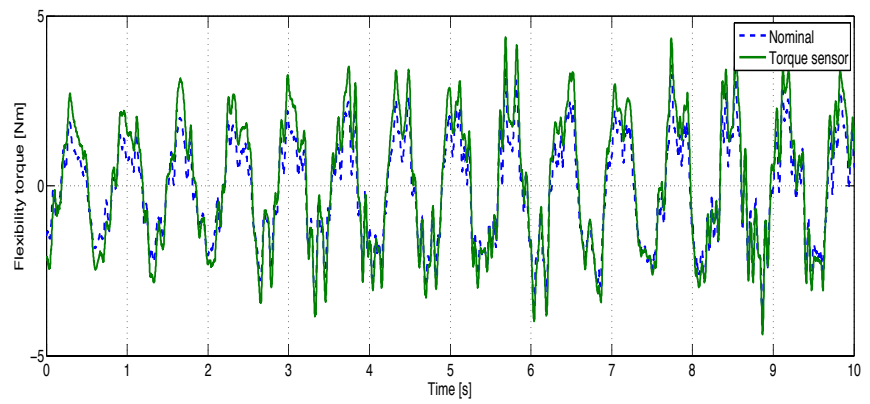

Fig. 11. Flexibility torque: nominal (dashed, blue), measured by the torque sensor (solid, green)

Figure 12 shows the stiffness estimation results. As in the simulations of Sect. VI-B, we consider three different estimation modalities: MODEL, SENSOR, and RESIDUAL. 


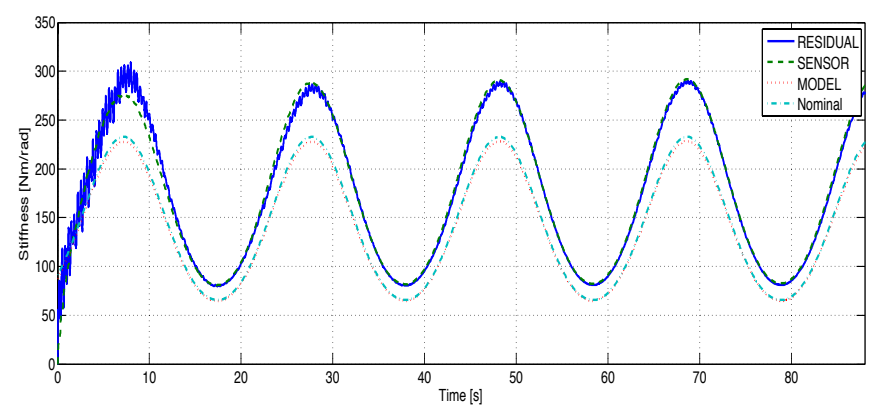

Fig. 12. Estimated stiffness: with nominal data (dot-dashed, green), with MODEL estimation method (dotted, red), with SENSOR estimation method (dashed, green), with RESIDUAL estimation method (solid, blue)

Before proceeding, it is worth noting that the nominal flexibility torque obtained using eq. (28) and the nominal AwAS data given in Sect. VI-A can be different from the real flexibility torque of the AwAS, due to unmodeled dynamics and uncertain knowledge of the model parameters. This will also be reflected in a difference between the real stiffness and the nominal one. In fact, the MODEL estimation method is very accurate in tracking the nominal stiffness (the two traces are practically superposed in Fig. 12). Indeed, the comparison of these two obtained results provides MSE = 9.36 and MSREP $=0.034 \%$ as performance indices. Stated differently, if the nominal stiffness had been the real one the MODEL estimation method would have worked properly. However, the results from the SENSOR estimation method, that we assume to provide the real ground truth stiffness, show a sensible difference with respect to the nominal stiffness and therefore the actual need for an independent stiffness estimator. On the other hand, the stiffness estimation by the RESIDUAL method is quite accurate in reproducing the SENSOR estimation results. Their relative comparison in terms of performance indices yields MSE $=63.02$ and MSREP $=1.55 \%$.

\section{CONCLUSIONS}

We have presented a robust stiffness estimator for robot joints with flexible transmissions, in particular for joints with a principal motor used to control link motion and a secondary motor used to separately adjust stiffness. The estimator is realized in two stages. The first stage estimates the flexibility torque of the transmission with a residual-based method, using only the parameters of the principal motor and its position sensor. In our discrete-time implementation, a modified kinematic Kalman filter reduces the effects of encoder discretization and quantization. The second stage completes the stiffness estimation based on a RLS algorithm, taking as inputs the transmission deformation, the secondary motor position, and the flexibility torque estimated in the first stage. The RLS algorithm has been enhanced so as to obtain convergence also under poor excitation conditions, which arise in serial configuration variable stiffness devices due to the limited transmission deformations involved. The role of the different components of the estimator and the robustness of the whole algorithm have been verified first by simulations. The presented experiments with the IIT AwAS joint have confirmed the necessity and the benefits of using the proposed stiffness estimator, which is able to compensate for uncertain parameters and unmodeled dynamics in the flexible transmission.

\section{REFERENCES}

[1] A. De Luca and W. Book, "Robots with flexible elements," in Springer Handbook of Robotics, B. Siciliano and O. Khatib, Eds. Springer, 2008, pp. 287-319.

[2] K. Ikuta, H. Ishii, and M. Nokata, "Safety evaluation method of design and control for human-care robots," Int. J. of Robotics Research, vol. 22, no. 7/8, pp. 281-297, 2003.

[3] A. De Santis, B. Siciliano, A. De Luca, and A. Bicchi, "An atlas of physical human-robot interaction," Mechanism and Machine Theory, vol. 43 , no. 3, pp. 253-270, 2008.

[4] S. Migliore, E. Brown, and S. DeWeerth, "Biologically inspired joint stiffness control," in Proc. IEEE Int. Conf. on Robotics and Automation, 2005, pp. 4508-4513.

[5] G. Tonietti, R. Schiavi, and A. Bicchi, "Design and control of a variable stiffness actuator for safe and fast physical human/robot interaction," in Proc. IEEE Int. Conf. on Robotics and Automation, 2005, pp. 528-533.

[6] R. Schiavi, G. Grioli, S. Sen, and A. Bicchi, "VSA-II: A novel prototype of variable stiffness actuator for safe and performing robots interacting with humans," in Proc. IEEE Int. Conf. on Robotics and Automation, 2008, pp. 2171-2176.

[7] A. De Luca, F. Flacco, A. Bicchi, and R. Schiavi, "Nonlinear decoupled motion-stiffness control and collision detection/reaction for the VSA-II variable stiffness device," in Proc. IEEE/RSJ Int. Conf. on Intelligent Robots and Systems, 2009, pp. 5487-5494.

[8] J. Choi, S. Park, W. Lee, and S.-C. Kang, "Design of a robot joint with variable stiffness," in Proc. IEEE Int. Conf. on Robotics and Automation, 2008, pp. 1760-1765.

[9] S. Wolf and G. Hirzinger, "A new variable stiffness design: Matching requirements of the next robot generation," in Proc. IEEE Int. Conf. on Robotics and Automation, 2008, pp. 1741-1746.

[10] Y. Ikegami, K. Nagai, R. Loureiro, and W. Harwin, "Design of redundant drive joint with adjustable stiffness and damping mechanism to improve joint admittance," in IEEE Int. Conf. on Rehabilitation Robotics (ICORR 2009), June 2009, pp. 202-210.

[11] A. Jafari, N. Tsagarakis, B. Vanderborght, and D. Caldwell, "A novel actuator with adjustable stiffness (AwAS)," in Proc. IEEE/RSJ Int. Conf. on Intelligent Robots and Systems, 2010, pp. 4201-4206.

[12] A. De Luca and F. Flacco, "Dynamic gravity cancellation in robots with flexible transmissions," in Proc. 49th IEEE Conf. on Decision and Control, 2010, pp. 288-295.

[13] A. Serio, G. Grioli, I. Sardellitti, N. G. Tsagarakis, and A. Bicchi, "A decoupled impedance observer for a variable stiffness robot," in Proc. IEEE Int. Conf. on Robotics and Automation, Shanghai, PRC, May 2011.

[14] G. Grioli and A. Bicchi, "A non-invasive real-time method for measuring variable stiffness," in Proc. Robotics Science and Systems (RSS 2010), Zaragoza, E, June 2010.

[15] _ , "A real-time parametric stiffness observer for vsa devices," in Proc. IEEE Int. Conf. on Robotics and Automation, Shangai, PRC, May 2011.

[16] F. Flacco and A. De Luca, "Residual-based stiffness estimation in robots with flexible transmissions," in Proc. IEEE Int. Conf. on Robotics and Automation, Shanghai, PRC, May 2011.

[17] _ - "Stiffness estimation and nonlinear control of robots with variable stiffness actuation," in 18th IFAC World Congr., Milano, I, Sep 2011.

[18] S. Bittanti, P. Bolzern, and M. Campi, "Recursive least-squares identification algorithms wit incomplete excitation: Convergence analysis and application to adaptive control," IEEE Trans. on Automatic Control, vol. 35, no. 12, pp. 1371-1373, 1990.

[19] R. Ozawa and H. Kobayashi, "Response characteristics of elastic joint robots driven by various types of controllers against external disturbances," in Proc. 6th Int. Conf. on Motion and Vibration Control, 2002, pp. 420-425. 